\title{
Spin Textures of Exciton-Polaritons in a Tunable Microcavity with Large TE-TM Splitting
}

\author{
S. Dufferwiel, ${ }^{1}$ Feng Li ${ }^{1,}{ }^{1}$ E. Cancellieri, ${ }^{1}$ L. Giriunas, ${ }^{1}$ A. A. P. Trichet, ${ }^{2}$ D. M. Whittaker, ${ }^{1}$ P. M. Walker, ${ }^{1}$ \\ F. Fras, ${ }^{1,3}$ E. Clarke, ${ }^{4}$ J. M. Smith, ${ }^{2}$ M. S. Skolnick, ${ }^{1}$ and D. N. Krizhanovskii ${ }^{1, \dagger}$ \\ ${ }^{1}$ Department of Physics and Astronomy, University of Sheffield, Sheffield S3 7RH, United Kingdom \\ ${ }^{2}$ Department of Materials, University of Oxford, Parks Road, Oxford OX1 3PH, United Kingdom \\ ${ }^{3}$ IPCMS UMR 7504, CNRS and Université de Strasbourg, 67200 Strasbourg, France \\ ${ }^{4}$ EPSRC National Centre for III-V Technologies, University of Sheffield, Sheffield S1 3JD, United Kingdom
}

(Received 9 March 2015; published 8 December 2015; corrected 10 February 2017)

\begin{abstract}
We report an extended family of spin textures of zero-dimensional exciton-polaritons spatially confined in tunable open microcavity structures. The transverse-electric-transverse-magnetic (TE-TM) splitting, which is enhanced in the open cavity structures, leads to polariton eigenstates carrying quantized spin vortices. Depending on the strength and anisotropy of the cavity confining potential and of the TE-TM induced splitting, which can be tuned via the excitonic or photonic fractions, the exciton-polariton emissions exhibit either spin-vortex-like patterns or linear polarization, in good agreement with theoretical modeling.
\end{abstract}

DOI: 10.1103/PhysRevLett.115.246401

Vortices are topological entities associated with quantized orbital angular momentum (OAM) which occur in many physical systems in optics, condensed matter, cosmology, and fundamental particles, characterized by a phase winding of an integer multiple of $2 \pi$ around a core. Structured light carrying OAM can be used in a broad range of applications including quantum information [1-3], topological photonics [4], optical forces [5], and vacuum slow light [6]. The coherent superposition of two modes with antirotating OAM and opposite photon pseudospin (circular polarization) is shown to lead to new types of topological entities, usually referred to as vector vortex beams in photonics [7-9] and spin vortices in excitonpolaritons $[10,11]$, characterized by quantized polarization winding instead of pure phase winding. Vector vortex lattices were reported in semiconductor lasers [12].

On the other hand, strong exciton-photon coupling in semiconductor microcavities leads to formation of polaritons. Much effort has been devoted to the development of methods to create OAM in a controllable way in a polariton system, such as optical imprinting [13,14] and chiral polaritonic lenses [15]. Meanwhile, quantized phase [16-18] and spin vortices [10] may also form spontaneously in exciton-polariton superfluids and nonequilibrium polariton Bose-Einstein condensates (BECs) subject to disorder potential $[10,16]$, although the exact origin of the latter remains unclear.

Published by the American Physical Society under the terms of the Creative Commons Attribution 3.0 License. Further distribution of this work must maintain attribution to the author(s) and the published article's title, journal citation, and DOI.
PACS numbers: 71.36.+c, 42.55.Sa, 71.70.Ej, 78.55.Cr

Another notable characteristic of semiconductor microcavities is the transverse-electric-transverse-magnetic (TE-TM) splitting [19], which defines two nondegenerate polarization directions relative to the in-plane wave vector [20]. In optical microcavities, TE-TM splitting enables the observation of interesting optical phenomena including the optical spin-Hall effect [21], magneticmonopole-like half solitons [22], spinor condensate with half-quantum circulation [23], and possibly topological insulators [24-26].

In this Letter we demonstrate the controlled realization of polaritonic spin vortices in an open-access microcavity with a tunable texture, where a top concave mirror creates a zero-dimensional confinement potential for polaritons. The large TE-TM splitting in the open cavity, which consists of two Bragg mirrors separated by an air gap, defines the polariton eigenstates described by an extended family of spin vortices and textures. We also observe polariton emissions showing both spin-vortex-like patterns as well as linearly polarized states depending on the interplay between the strength and the anisotropy of the confining potential and the strength of the TE-TM splitting, which can be modified by changing the exciton or photon fraction. In this work polariton condensation makes the spin patterns spectrally visible due to increased polariton temporal coherence above the condensation threshold. We note that the observed spin textures, being determined by the eigenstates of the cavity, should be distinguished from vortices reported in extended BECs and superfluids, which are collective states arising from interparticle interactions $[27,28]$.

The open microcavity system consists of planar bottom distributed Bragg reflectors (DBR) and a concave top DBR 


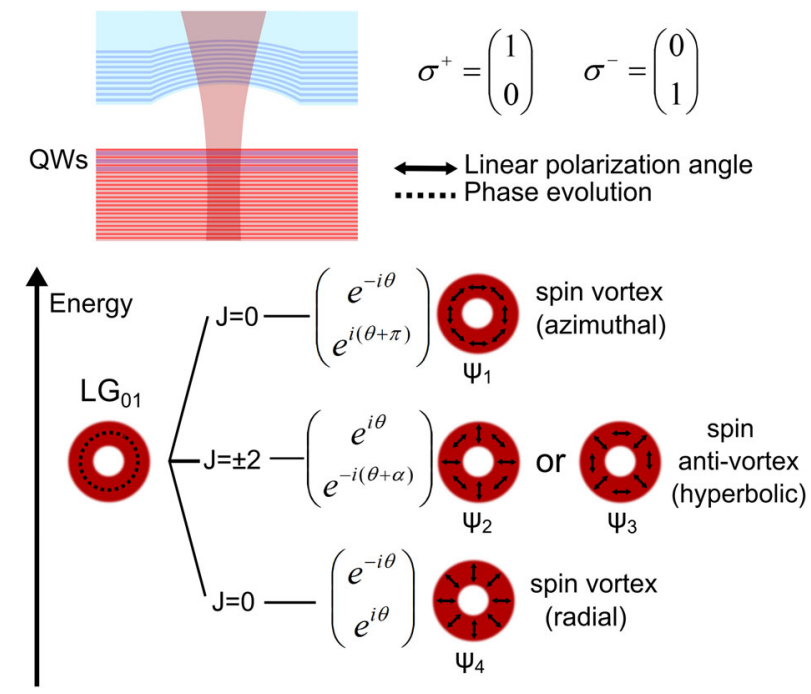

FIG. 1 (color online). Illustrative graph of the new eigenstates formed by $\mathrm{LG}_{01}$ mode due to TE-TM splitting. The TE-TM splitting in the cavity lifts the degeneracy of the modes leading to three energy levels characterized by spin vortices and antivortices. The mathematical form of the azimuthal part of each eigenstate is labeled next to its illustrative diagram. The top-left inset is a sketch of the open cavity. The basis of circular polarization is defined in the top-right inset.

[29] controlled independently by nanopositioners (top-left inset of Fig. 1), which allows free tuning of the spectral resonance by changing the mirror separation [30]. A total number of $12 \mathrm{GaAs}$ quantum wells (QWs) are grown above the surface of the bottom DBR at electric field antinodes, allowing the strong exciton-cavity coupling regime to be reached with a Rabi splitting of $\sim 15 \mathrm{meV}$ [29]. Polariton condensation is demonstrated with a nonlinear increase of emission intensity, sharp linewidth reduction, and a small blueshift $(\sim 1 \mathrm{meV})$ far below the bare cavity mode at $\sim 6 \mathrm{meV}$ to higher energy [29].

The top concave mirror induces a strong and almost harmonic lateral confinement of polaritons [30], and therefore the effect of TE-TM splitting or the asymmetries in the circular shape of the top mirror can be studied perturbatively [29]. In order to fully describe the eigenmodes of the system two bases of Laguerre-Gauss modes can be used, one for each pseudospin component: $L G_{p l}^{\sigma^{ \pm}}$, where $\sigma^{+}\left(\sigma^{-}\right)$represent polaritons associated with left (right) circularly polarized light, and $p$ and $l$ are quantum numbers quantifying the radial and azimuthal phase evolution, respectively.

We consider the simplest case of perfectly circular mirrors. Since only Laguerre-Gauss modes with $l \neq 0$ carry nonzero $\mathrm{OAM} \pm \hbar l$, corresponding to a phase rotation of $2 \pi l$ either clockwise or anti-clockwise, our analysis starts from the first excited manifold (FEM) of the harmonic potential $\mathrm{LG}_{0 \pm 1}^{\sigma_{ \pm}}$, without considering the effect of TE-TM splitting:

$$
\begin{aligned}
\mathrm{LG}_{01}^{\sigma^{+}} & =C(r) \varphi_{11}(\theta)=C(r)\left(\begin{array}{c}
e^{i \theta} \\
0
\end{array}\right), \\
\mathrm{LG}_{01}^{\sigma^{-}} & =C(r) \varphi_{-11}(\theta)=C(r)\left(\begin{array}{c}
0 \\
e^{i \theta}
\end{array}\right), \\
\mathrm{LG}_{0-1}^{\sigma^{+}} & =C(r) \varphi_{1-1}(\theta)=C(r)\left(\begin{array}{c}
e^{-i \theta} \\
0
\end{array}\right), \\
\mathrm{LG}_{0-1}^{\sigma^{-}} & =C(r) \varphi_{-1-1}(\theta)=C(r)\left(\begin{array}{c}
0 \\
e^{-i \theta}
\end{array}\right),
\end{aligned}
$$

where $\varphi_{s l}(\theta)$ is the azimuthal part of the polariton wave function with $s= \pm 1$ corresponding to polaritons associated with $\sigma^{ \pm}$polarized light, $C(r)$ is the radial part of the normalized Laguerre-Gauss mode with $l= \pm 1$, and $\theta$ and $r$ are angular and radial coordinates. The total angular momentum can be defined for each $\varphi_{s l}(\theta)$ as $J=l+s$, being $J=-2,0$ or +2 . Using degenerate perturbation theory and including the TE-TM splitting term, one obtains the following new eigenmodes [29]:

$$
\begin{aligned}
& \psi_{1}(r, \theta)=\frac{1}{\sqrt{2}} C(r)\left[\varphi_{1-1}(\theta)+\varphi_{-11}(\theta+\pi)\right], \\
& \psi_{2}(r, \theta)=\frac{1}{\sqrt{2}} C(r)\left[\varphi_{11}(\theta)+\varphi_{-1-1}(\theta],\right. \\
& \psi_{3}(r, \theta)=\frac{1}{\sqrt{2}} C(r)\left[\varphi_{11}(\theta)+\varphi_{-1-1}(\theta-\pi)\right], \\
& \psi_{4}(r, \theta)=\frac{1}{\sqrt{2}} C(r)\left[\varphi_{1-1}(\theta)+\varphi_{-11}(\theta)\right],
\end{aligned}
$$

with eigenenergies: $E_{1}=E_{0}+2 \beta / \sigma^{2}, E_{2}=E_{3}=E_{0}$, and $E_{4}=E_{0}-2 \beta / \sigma^{2}$, where $E_{0}$ is the energy of the $\mathrm{LG}_{01}$ mode, $\beta=\hbar^{2}\left(1 / m_{\mathrm{TE}}-1 / m_{\mathrm{TM}}\right) / 4$ is a parameter related to the TE-TM splitting $\left[m_{\mathrm{TE}(\mathrm{TM})}\right.$ are the lower-polariton masses in the TE(TM) polarizations], and $\sigma$ is the parameter defining the size of the modes [29]. The structure of the new eigenmodes, being spin vortices as illustrated in Fig. 1, can be understood by observing that the TE-TM splitting lifts the degeneracy by coherently combining the $J=0$ wave functions $\left(\varphi_{1-1}\right.$ and $\left.\varphi_{-11}\right)$ to form new eigenstates, while leaving the energy of the two $J= \pm 2$ modes unaffected. For the $J= \pm 2$ modes any linear combination of $\varphi_{11}$ and $\varphi_{-1-1}$ is a suitable eigenmode in the presence of TE-TM splitting. Similarly to the case of spin vortices in planar two-dimensional cavities [11], here we obtain that the splitting between the new eigenmodes is linearly dependent on $\beta$.

To investigate the properties of spin vortices, low temperature photoluminescence (PL) measurements were carried out (details in Ref. [29]). In the first set of measurements, a concave mirror with a radius of curvature (ROC) of $20 \mu \mathrm{m}$ was employed and the mirror separation was $\sim 1 \mu \mathrm{m}$. The cavity is detuned so that polaritons in the FEM modes have a photonic fraction of $\sim 64 \%$. Below the condensation threshold, the spectrum associated with 

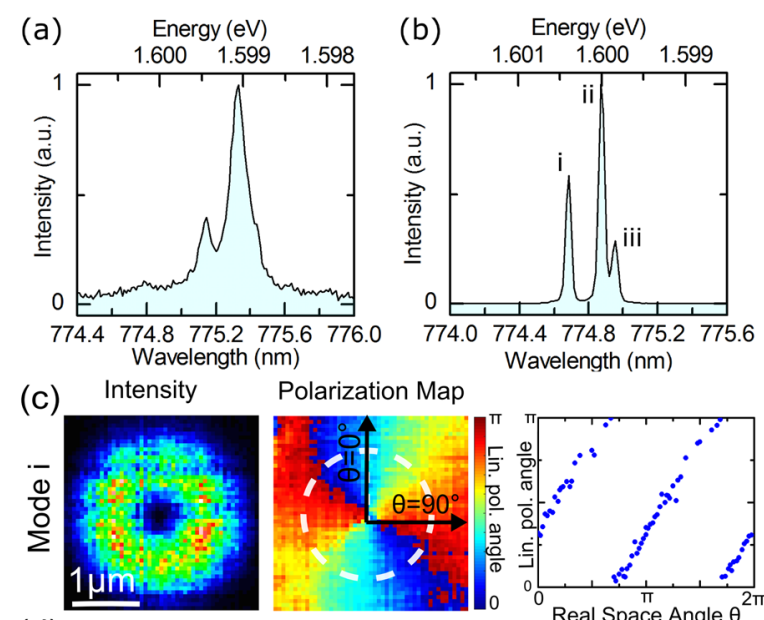

Wavelength $(\mathrm{nm})$
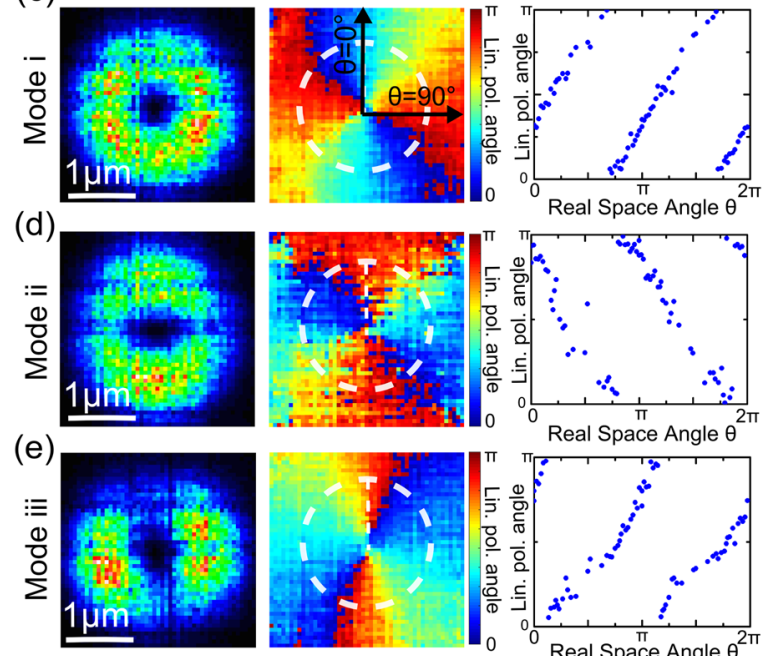

Real Space Angle $\theta$

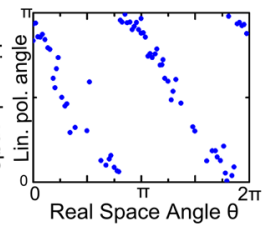

Real Space Angle $\theta$

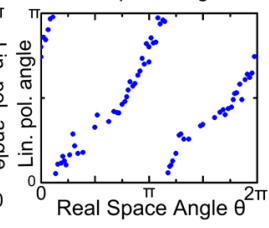

FIG. 2 (color online). PL spectra of the FEM below threshold (a) and above threshold (b) where linewidth narrowing allows the resolution of three different modes. The ROC of the top concave mirror is $20 \mu \mathrm{m}$ and the photonic fraction is 64\%. (c)-(e) Spatial intensity and polarization properties of mode i (c), ii (d), and iii (e). The left panels show the real space PL intensity; the middle panels show the real space distribution of the linear polarization angle; the right panels shows the linear polarization angle as a function of the real space winding angle circulating clockwise around the white dashed circle in the middle panel. $0^{\circ}$ and $90^{\circ}$ are defined in the middle panel of (c), which also defines the spatial coordinates used for all figures in this Letter.

the FEM displays two broad features, as shown in Fig. 2(a). With increase of pump power, condensation occurs and the linewidths drop sharply due to an increase of temporal coherence. Three well-resolved modes labeled by i, ii, and iii are now revealed in Fig. 2(b). Energy resolved images, shown in the left panels of Figs. 2(c), 2(d), and 2(e), show a ringlike field distribution for all the three modes. The imperfection of the ring shape of mode iii is due to slight asymmetry of the confinement potential as will be discussed later. A linear polarizer and a quarter wave plate are inserted into the optical path to collect polarization and energy resolved images for each mode in the horizontalvertical $\left(0^{\circ}\right.$ and $\left.90^{\circ}\right)$ basis, diagonal $\left( \pm 45^{\circ}\right)$ basis, and circular $\left(\sigma^{+}\right.$and $\left.\sigma^{-}\right)$basis, and the associated Stokes parameters, $S_{1}, S_{2}$, and $S_{3}$, are calculated for each pixel of the image [29]. The linear polarization angle $\phi$, defined as $2 \phi=\arctan \left(S_{2} / S_{1}\right)$, is mapped out for each mode in the middle panels of Figs. 2(c), 2(d), and 2(e). As the circular polarization degree $\left(S_{3}\right)$ is low for all the three modes [29], the linear polarization vectors characterize well the spin textures.

All three modes display quantized pseudospin currents characterized by a $2 \pi$ rotation of $\phi$ around the mode cores, with a high linear polarization degree $\sqrt{S_{1}^{2}+S_{2}^{2}} \sim 0.95$ being exhibited. For both modes i and iii, $\phi$ changes nearly linearly with the real space azimuthal angle $\theta$, corresponding to the rotation of the vector of linear polarization clockwise around the mode center, which indicates a corotating relation between $\theta$ and $\phi$, as indicated by the right panels of Figs. 2(c) and 2(e). At $\theta=0^{\circ}$, we observe $\phi=\sim 90^{\circ}$ (horizontal polarization) for mode $\mathrm{i}$ and $\phi=\sim 0^{\circ}$ (vertical polarization) for mode iii $\left[0^{\circ}\right.$ is defined as vertical, see the middle panel of Fig. 2(c)], showing they are azimuthal and radial spin vortices corresponding to the TE and TM eigenmodes $\psi_{1}$ and $\psi_{4}$ in Fig. 1, respectively. By contrast, mode ii is a spin antivortex displaying the opposite pseudospin vector rotation with respect to $\mathrm{i}$ and iii, with $\phi$ and $\theta$ counterrotating [right panel Fig. 2(d)]. Its hyperboliclike polarization pattern results from the coherent combination, with any initial phase difference, of $J= \pm 2$ states with different polarizations (modes $\psi_{2}$ and $\psi_{3}$ in Fig. 1 correspond to the case with a phase difference of 0 or $\pi$ ). The energy splitting of $0.56 \mathrm{meV}$ observed between modes i and iii, along with $\sigma=0.65 \mu \mathrm{m}$ obtained from the actual sizes of the spin vortices, indicates a TE-TM splitting factor $\beta=0.06 \mathrm{meV} \mu \mathrm{m}^{2}$ [29], a value $\sim 3$ times larger than that reported in monolithic microcavities $[21,22,29,31]$. The large value of $\beta$ mainly arises from the phase shifts due to reflections at the air gap interfaces in the open cavity system. Possible reasons for the unequal energy spacing between modes $\mathrm{i}$, ii, and iii are discussed in Ref. [29].

We also observe spin textures for polaritons condensed into higher order LG-associated modes, when these are tuned into resonance with the exciton. Similar to the $\mathrm{LG}_{0 \pm 1}$ case, TE-TM splitting also mixes modes in the second excited manifold (SEM) like, for example, $\mathrm{LG}_{10}$ and $\mathrm{LG}_{02}$ modes [38]. As illustrated in Fig. 3(a), the modes formed are quasi-spin-vortices labeled as type $A$ and $B$. The polarization vectors exhibit radial $(A)$ or azimuthal $(B)$ spin vortex character in the inner core and azimuthal $(A)$ or radial $(B)$ spin vortex character in the outer ring, connected by transient elliptically polarized states. Such quasi-spinvortices were experimentally observed as shown in Figs. 3(b) and 3(c), with a change of linear polarization angle of $\pi$ between the inner core and outer ring. Here above condensation threshold four spectrally resolved condensates are observed and for simplicity we show polarization patterns only for two of them, which fully demonstrate the principle illustrated in Fig. 3(a). The imperfection of the mode spatial profile and the linearlike 
(a)
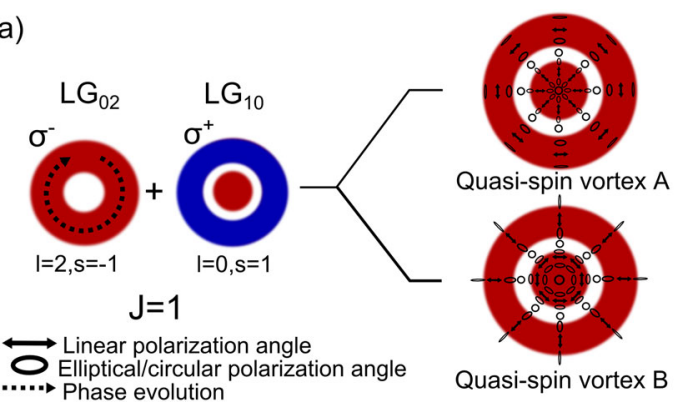

(b) Intensity

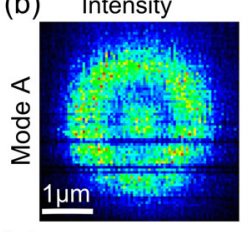

(c)

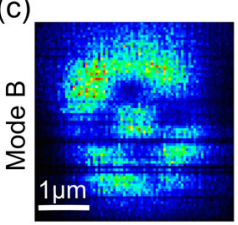

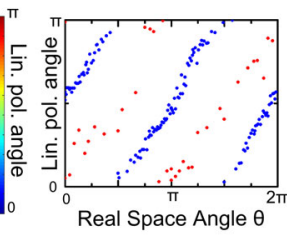

Real Space Angle $\theta$

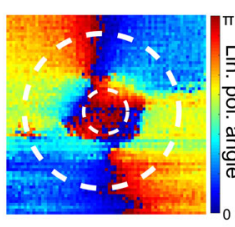

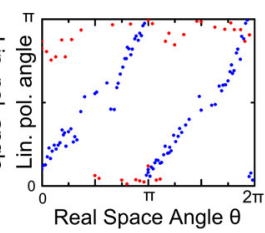

FIG. 3 (color online). (a) Schematics of the generation of spin textures with higher order LG modes. (b) and (c) Experimental observation of the nontrivial spin modes in (a), with left, middle, and right panels showing intensity profiles, linear polarization angle maps, and polarization angle winding. The blue and red traces in the right-hand panels show the outer and inner rotations defined by the dashed white circles in the middle panel. The horizontal dark lines on (b) and (c) arise from dark pixels on the CCD detector.

polarization vector of the inner core in Fig. 3(c) compared to 3(a) are most likely due to the slightly elliptical shape of the top concave mirror.

If the concave top mirror has a sufficiently strong ellipticity which perturbs the harmonic confinement potential along the two orthogonal directions with strength $a$ (see Ref. [29] for details) it may induce Mathieu-Gauss (MG) modes $[39,40]$ which are characterized by linearly polarised orthogonal double-lobe profiles [see Fig. 4(d)]. The eigenmodes of the cavity arise from the competition between the asymmetry of the mirror and the strength of the TE-TM splitting: either spin vortices or linear polarized states will be formed depending on which term dominates. In order to achieve condensation in MG modes the TE-TM splitting can be reduced by tuning the energy of the condensed modes closer to the exciton, and by using mirrors with smaller ROC, where the confinement potential is stronger and the spatial anisotropy is more pronounced.

From these considerations a concave mirror with a ROC of $7 \mu \mathrm{m}$ is chosen, and a photon fraction of $41 \%$ employed. Figure 4(a) shows the spectrum of the polariton condensate associated with the FEM, where the low energy modes ii and iii are preferentially selected above threshold, leading

(a)

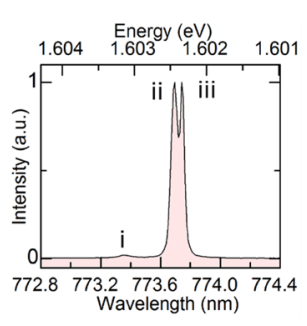

(b) Intensity

Polarization Map
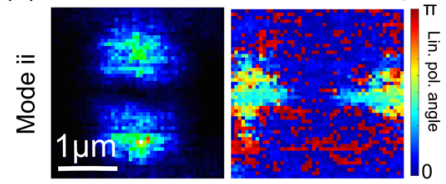

(c)

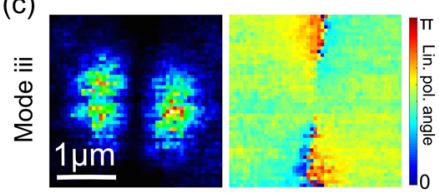

(d)
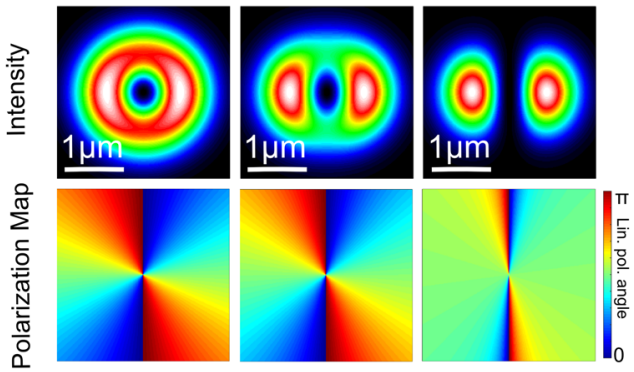

FIG. 4 (color online). (a) Spectrum of the FEM above threshold with a photonic fraction of $41 \%$ for a top concave mirror with ROC $=7 \mu \mathrm{m}$. (b),(c) Real-space PL intensity profile (left panel) and real-space distribution of linear polarization angle derived from the Stokes parameters for modes ii and iii (right panel). (d) Simulation demonstrating the impact on the spin textures of the interplay between TE-TM splitting and the cavity ellipticity. The ellipticity parameter $a$ is set to $-0.05 \mathrm{meV} \mu \mathrm{m}^{2}$ for all graphs. Upper panels show real-space intensity profiles for TE-TM splitting factors $\beta=0.09,0.02$, and $0.002 \mathrm{meV} \mu \mathrm{m}^{2}$ from left to right, while the lower panels show the corresponding real-space maps of the polarization angle. When the TE-TM splitting decreases the mode changes gradually from a spin vortex to a linear polarized MG-like profile. The definition of $\beta$ and $a$ is detailed in the Supplemental Material [29].

to significantly larger intensity than mode i. Nontrivial differences, compared to the spin vortices in Fig. 2, are found in the mode spatial profiles and polarization patterns, as shown in Figs. 4(b) and 4(c) for mode ii and iii. Instead of being spin vortices or antivortices, modes ii and iii clearly show MG-like orthogonal double-lobe profiles (left panels) with vertical (ii) and horizontal (iii) linear polarization (right panels). The simulated intensity distribution and polarization maps of one of the eigenstates confined in an elliptical potential are shown in Fig. 4(d) for decreasing TE-TM splitting factors. Theoretically, it is seen that smaller TE-TM splitting leads to the MG mode being the eigenstates of the system as the ellipticity term has greater influence. Importantly, for the same ROC $=7 \mu \mathrm{m}$ mirror, we can recover the vortexlike spin textures for all three modes similar to those shown in Fig. 2 by doubling the photonic fraction up to $82 \%$, as shown in the Supplemental Material [29]. In addition, this enhancement of the TE-TM splitting increasing the photon fraction results in increase of the $\mathrm{i}-\mathrm{ii}$ mode splitting from 0.71 to 
$1.02 \mathrm{meV}$. This demonstrates the advantages of the tunability of the open cavities in permitting the degree of the light and matter fractions of the cavity polaritons to be varied but also in allowing flexible manipulation of the polarization textures.

In summary, we have demonstrated polariton emission exhibiting spin vortices and more elaborate spin textures in a tunable microcavity system with lateral confinement. These spin textures can be described as orthogonal eigenstates on the higher order Poincare sphere (HOPS) [41]. This might lead to a new type of cavity quantum eletrodynamics by manipulating the vector states on the HOPS involving both the OAM and polarization (pseduospin) degrees of freedom, since the excitonic part of the polaritons can be dynamically manipulated with external magnetic field or fast Stark pulses [42,43].

We acknowledge support by EPSRC Grant No. EP/ J007544, ERC Advanced Grant EXCIPOL, and the Leverhulme Trust Grant No. RPG-2013-339.

Note added.-Recently, spin vortex phenomena in polariton micropillars coupled in a hexagonal pattern were reported in Ref. [44].

*Corresponding author.

f.li@sheffield.ac.uk

†d.krizhanovskii@sheffield.ac.uk

[1] A. Mair, A. Vaziri, G. Weihs, and A. Zeilinger, Nature (London) 412, 313 (2001).

[2] E. Nagali, F. Sciarrino, F. De Martini, L. Marrucci, B. Piccirillo, E. Karimi, and E. Santamato, Phys. Rev. Lett. 103, 013601 (2009).

[3] R. Fickler, R. Lapkiewicz, M. Huber, M. P. Lavery, M. J. Padgett, and A. Zeilinger, Nat. Commun. 5, 4502 (2014).

[4] T. Bauer, P. Banzer, E. Karimi, S. Orlov, A. Rubano, L. Marrucci, E. Santamato, R. W. Boyd, and G. Leuchs, Science 347, 964 (2015).

[5] D. B. Ruffner and D. G. Grier, Phys. Rev. Lett. 108, 173602 (2012).

[6] D. Giovannini, J. Romero, V. Potoček, G. Ferenczi, F. Speirits, S. M. Barnett, D. Faccio, and M. J. Padgett, Science 347, 857 (2015).

[7] F. Prati, G. Tissoni, M. San Miguel, and N. Abraham, Opt. Commun. 143, 133 (1997).

[8] F. Cardano, E. Karimi, S. Slussarenko, L. Marrucci, C. de Lisio, and E. Santamato, Appl. Opt. 51, C1 (2012).

[9] L. Gong, Y. Ren, W. Liu, M. Wang, M. Zhong, Z. Wang, and Y. Li, J. Appl. Phys. 116, 183105 (2014).

[10] F. Manni, Y. Léger, Y. G. Rubo, R. André, and B. Deveaud, Nat. Commun. 4, 2590 (2013).

[11] M. Toledo-Solano, M. E. Mora-Ramos, A. Figueroa, and Y. G. Rubo, Phys. Rev. B 89, 035308 (2014).

[12] Y. F. Chen, K. F. Huang, H. C. Lai, and Y. P. Lan, Phys. Rev. Lett. 90, 053904 (2003).

[13] D. N. Krizhanovskii, D. M. Whittaker, R. A. Bradley, K. Guda, D. Sarkar, D. Sanvitto, L. Vina, E. Cerda, P. Santos,
K. Biermann, R. Hey, and M. S. Skolnick, Phys. Rev. Lett. 104, 126402 (2010).

[14] T. Boulier, E. Cancellieri, N. D. Sangouard, Q. Glorieux, D. M. Whittaker, E. Giacobino, and A. Bramati, arXiv: 1509.02680 .

[15] R. Dall, M. D. Fraser, A. S. Desyatnikov, G. Li, S. Brodbeck, M. Kamp, C. Schneider, S. Höfling, and E. A. Ostrovskaya, Phys. Rev. Lett. 113, 200404 (2014).

[16] K. G. Lagoudakis, M. Wouters, M. Richard, A. Baas, I. Carusotto, R. Andre, L. S. Dang, and B. Deveaud-Pledran, Nat. Phys. 4, 706 (2008).

[17] G. Nardin, G. Grosso, Y. Léger, B. Piętka, F. Morier-Genoud, and B. Deveaud-Plédran, Nat. Phys. 7, 635 (2011).

[18] D. Sanvitto, S. Pigeon, A. Amo, D. Ballarini, M. De Giorgi, I. Carusotto, R. Hivet, F. Pisanello, V. G. Sala, P. S. S. Guimaraes, R. Houdre, E. Giacobino, C. Ciuti, A. Bramati, and G. Gigli, Nat. Photonics 5, 610 (2011).

[19] G. Panzarini, L. C. Andreani, A. Armitage, D. Baxter, M. S. Skolnick, V. N. Astratov, J. S. Roberts, A. V. Kavokin, M. R. Vladimirova, and M. A. Kaliteevski, Phys. Rev. B 59, 5082 (1999).

[20] A. Kavokin, G. Malpuech, and M. Glazov, Phys. Rev. Lett. 95, 136601 (2005).

[21] C. Leyder, M. Romanelli, J. P. Karr, E. Giacobino, T. C. H. Liew, M. M. Glazov, A. V. Kavokin, G. Malpuech, and A. Bramati, Nat. Phys. 3, 628 (2007).

[22] R. Hivet, H. Flayac, D. D. Solnyshkov, D. Tanese, T. Boulier, D. Andreoli, E. Giacobino, J. Bloch, A. Bramati, G. Malpuech, and A. Amo, Nat. Phys. 8, 724 (2012).

[23] G. Liu, D. W. Snoke, A. Daley, L. N. Pfeiffer, and K. West, Proc. Natl. Acad. Sci. U.S.A. 112, 2676 (2015).

[24] L. Lu, J. D. Joannopoulos, and M. Soljačić, Nat. Photonics 8, 821 (2014).

[25] A. V. Nalitov, D. D. Solnyshkov, and G. Malpuech, Phys. Rev. Lett. 114, 116401 (2015).

[26] C.-E. Bardyn, T. Karzig, G. Refael, and T. C. H. Liew, Phys. Rev. B 91, 161413 (2015).

[27] L. E. Sadler, J. M. Higbie, S. R. Leslie, M. Vengalattore, and D. Stamper-Kurn, Nature (London) 443, 312 (2006).

[28] M. R. Matthews, B. P. Anderson, P. C. Haljan, D. S. Hall, C. E. Wieman, and E. A. Cornell, Phys. Rev. Lett. 83, 2498 (1999).

[29] See Supplemental Material at http://link.aps.org/ supplemental/10.1103/PhysRevLett.115.246401 for theoretical models and experimental details, which includes Refs. [13,21,22,30,31,39-44].

[30] S. Dufferwiel, F. Fras, A. Trichet, P. M. Walker, F. Li, L. Giriunas, M. N. Makhonin, L. R. Wilson, J. M. Smith, E. Clarke, M. S. Skolnick, and D. N. Krizhanovskii, Appl. Phys. Lett. 104, 192107 (2014).

[31] H. Flayac, D. D. Solnyshkov, I. A. Shelykh, and G. Malpuech, Phys. Rev. Lett. 110, 016404 (2013).

[32] P. R. Dolan, G. M. Hughes, F. Grazioso, B. R. Patton, and J. M. Smith, Opt. Lett. 35 (2010).

[33] E. Wertz, L. Ferrier, D. D. Solnyshkov, R. Johne, D. Sanvitto, A. Lemaitre, I. Sagnes, R. Grousson, A. V. Kavokin, P. Senellart, G. Malpuech, and J. Bloch, Nat. Phys. 6, 860 (2010). 
[34] D. Bajoni, P. Senellart, E. Wertz, I. Sagnes, A. Miard, A. Lemaître, and J. Bloch, Phys. Rev. Lett. 100, 047401 (2008).

[35] J. Kasprzak, D. D. Solnyshkov, R. André, L. S. Dang, and G. Malpuech, Phys. Rev. Lett. 101, 146404 (2008).

[36] F. Li, L. Orosz, O. Kamoun, S. Bouchoule, C. Brimont, P. Disseix, T. Guillet, X. Lafosse, M. Leroux, J. Leymarie, M. Mexis, M. Mihailovic, G. Patriarche, F. Réveret, D. Solnyshkov, J. Zuniga-Perez, and G. Malpuech, Phys. Rev. Lett. 110, 196406 (2013).

[37] H. Flayac, D. D. Solnyshkov, and G. Malpuech, Phys. Rev. B 83, 193305 (2011).

[38] D. H. Foster and J. U. Nöckel, Opt. Lett. 29, 2788 (2004).

[39] J. C. Gutierrez-Vega, R. M. Rodriguez-Dagnino, M. A. Meneses-Nava, and S. Chavez-Cerda, Am. J. Phys. 71, 233 (2003).
[40] G. Nardin, Y. Léger, B. Pietka, F. Morier-Genoud, and B. Deveaud-Plédran, Phys. Rev. B 82, 045304 (2010).

[41] G. Milione, H. I. Sztul, D. A. Nolan, and R. R. Alfano, Phys. Rev. Lett. 107, 053601 (2011).

[42] A. Hayat, C. Lange, L. A. Rozema, A. Darabi, H. M. van Driel, A. M. Steinberg, B. Nelsen, D. W. Snoke, L. N. Pfeiffer, and K.W. West, Phys. Rev. Lett. 109, 033605 (2012).

[43] E. Cancellieri, A. Hayat, A. M. Steinberg, E. Giacobino, and A. Bramati, Phys. Rev. Lett. 112, 053601 (2014).

[44] V. G. Sala, D. D. Solnyshkov, I. Carusotto, T. Jacqmin, A. Lemaître, H. Terças, A. Nalitov, M. Abbarchi, E. Galopin, I. Sagnes, J. Bloch, G. Malpuech, and A. Amo, Phys. Rev. X 5, 011034 (2015). 